\title{
Robust Control of Robot Manipulator based on QFT and $\mathrm{H}_{\infty}$
}

\author{
J. J. Carreño, R. Villamizar \\ Member, IEEE \\ Electrical Engineering Department, Universidad Industrial de Santander, \\ Cra 27 \# 9 Bucaramanga, Colombia
}

Received: April 18, 2021. Revised: Novemeber 12, 2021. Accepted: December 23, 2021. Published: January 9, 2022.

\begin{abstract}
Robust controllers have been developed by both control techniques QFT and $H_{\infty}$ applied in the waist, shoulder and elbow of a manipulator of 6 degrees of freedom. The design is based on the identification of a linear model of the robot dynamics which represents the non-linearity of the system using parametric uncertainty. QFT control methodology is used to tune the robust PID-controller and pre-filters of the system, and $\mathbf{H}_{\infty}$ controllers are obtained by designing the weighting functions and using the MATLAB hinfopt tool. Finally the performance of robust controllers is compared designed based on the calculation and analysis of some behavioral indices.
\end{abstract}

Keywords-robot dynamics; robust control; robustness; uncertainty

\section{INTRODUCTION}

A DVANCES in computing, communications and electronics have allowed that robotics and related technologies such as cybernetics and mechatronics may have an amazing growth in last decades. For this reason, in recent years the concept of robot has gone from a science fiction movie to become a reality as a s tandalone machine, with great potential for use in applications, since welding robots in the automotive industry, robots used in medicine for the study of the human body, until teleoperated arms in space shuttles, among others [1].

The main advantages supported for using this type of mechanism in the industry include reducing the production costs due to the increase of accuracy, productivity, quality, and flexibility compared to specialized machines. Therefore, the objective of the control system in robot manipulators is to maximize the accuracy, repeatability and speed of execution of tasks, taking into account the physical limitations of the actuators and establishing a commitment to the needs of each application practice.

In order to ensure an efficient dynamic performance in a robotic manipulator with nonlinearity conditions, coupled dynamics, disturbances and unmodeled dynamics, this paper proposes to use two robust control strategies that take into account these characteristics: Quantitative Feedback Theory (QFT) proposed by Horowitz [2] and the design of controllers using $H_{\mathrm{m}}$ technique [3].

Quantitative Feedback Theory has proved a very powerful methodology for the design of feedback systems controllers. Its effectiveness is due to its ability to tune robust controllers for systems where the plant presents uncertainty and / or there are disturbances acting on the plant. In the case of MIMO systems, as in the field of robotics, the classical idea of the QFT technique is based on fixed point theory wherein the multivariable system is broken down into single loop MISO systems and coupling effects are treated as interference to the entrance of the plant [4].

The $H_{m}$ control theory proposed by Zames in 1981, is widely recognized as an indispensable method of designing robust control systems. The optimum design $H_{m}$ is based on the minimization of an objective function specific to the dynamic constraints of the system, so that the controller meets the design objectives proposed. This approach has been widely discussed for both its stability robustness and disturbance attenuation capability in linear control systems and nonlinear time-invariant systems [5].

Motivated by the above discussion, PID controllers design based on control theory QFT and the design of $H_{m}$ controllers is proposed for a robotic system with parametric uncertainty. Finally the performance of robust controllers is compared designed based on the calculation and analysis of some behavioral indices.

This paper is organized as follows. In Section II, the model description of the manipulator and the model with parameter uncertainty are given. Section III presents the design of QFT and $H_{\mathrm{m}}$ controllers. In Section IV, simulations are performed to confirm the robust performances of the proposed controllers for robot manipulator under parameter uncertainty. In Section V, the conclusions are presented.

\section{DYNAMIC MODEL OF THE MANIPULATOR}

\section{A. Robot Dynamics}

According LaGrange's theory [1], [6], the dynamics of a manipulator with $n$-degrees-of-freedom is represented by:

$\tau=\mathrm{M}(\mathrm{q}) * \ddot{\mathrm{q}}+\mathrm{V}(\mathrm{q}, \dot{\mathrm{q}}) * \dot{\mathrm{q}}+\mathbf{F}(\dot{\mathrm{q}})+\mathrm{G}(\mathbf{q})+\tau_{\mathrm{p}}$

Where: 
M(q): Inertia matrix [Kg-rad], of dimensions $n \times n$.

$\mathbf{V}(\mathbf{q}, \mathbf{q})$ : Centrifugal and Coriolis forces matrix $[\mathrm{Kg}-$ $\mathrm{rad} / \mathrm{s}]$, of dimensions $\mathbf{n} \times \mathbf{1}$.

$\mathbf{F}(\mathbf{q}): \mathbf{n} \times \mathbf{1}$ vector modeling joint friction in $[\mathrm{N}]$.

$\mathrm{G}(\mathrm{q}): \mathbf{n} \times \mathbf{1}$ Gravitational torques vector in $\left[\mathrm{N}^{*} \mathrm{~m}\right]$.

$\tau_{\mathrm{p}}$ Disturbance that considers unmodeled dynamics of the system in $\left[\mathrm{N}^{*} \mathrm{~m}\right]$.

$\tau$ : Generalized force vector joints in $\left[\mathrm{N}^{*} \mathrm{~m}\right]$.

Unmodeled dynamics, meanwhile, are included in the pair of joint ${ }^{\tau_{p}}$ [7], which contains all types of external action. In regard to the actuator [8], it is a DC motor with negligible inductance which will result in $n$ uncoupled equations [9], one for each joint:

$$
\begin{aligned}
& \mathrm{u}-\mathrm{k}_{\mathrm{b}} \cdot \boldsymbol{q}_{\mathrm{m}}=\mathrm{R}_{\mathrm{a} \times} \mathrm{i} \\
& \tau_{m}=J_{m} \cdot \ddot{q}_{m}+B \cdot \dot{q}_{m}+R \cdot \tau \\
& \tau_{\mathrm{m}}=\mathbf{K} . \mathbf{i}
\end{aligned}
$$

Given that the following expression is obtained:

$$
\mathrm{I}_{\mathrm{m}} \cdot \overline{\mathrm{q}}_{\mathrm{m}}+\left(\mathrm{B}+\frac{\mathrm{K}_{\mathrm{v}} \mathrm{K}_{\mathrm{b}}}{\mathrm{R}_{\mathrm{a}}}\right) \cdot \dot{\mathrm{q}}+\mathrm{R} \cdot \tau=\mathrm{K}_{\mathrm{m}} \cdot \mathrm{u}
$$

Where in this case the articular variables shown represent positions, velocities and accelerations of rotation of the corresponding motor shaft [8], while $\mathbf{F}$ is a multiplicative term reduction due to coupling between the actuator and the manipulator.. Because of these reducers, it is obtained that $\mathrm{q}_{\mathbf{i}}=$ R. $\mathbf{q i}$

, where $\mathbf{q}_{\mathbf{i}}$ joint variables of the

manipulator and $\mathbf{q}_{\mathbf{m i}}$ represent the motor joint variables. Thus, introducing the dynamic equation of the manipulator in the motor equation, is obtained:

$$
J_{m} \cdot \bar{q}+B_{m} \cdot \dot{q}+R^{2} \tau=K_{m} \cdot R \cdot u
$$

However, considering the large amount of reducers it can perform the next approximation to obtain a linear model uncertainty:

$$
J_{e f} \cdot q+B_{e f} \cdot q+\tau_{d}=K_{R} u
$$

Where $\tau_{\mathrm{d}}$ is the disturbance due to the system performance and other friction joints herein, and secondly

. Thus, as shown in Figure 1 the system has a linear model of each joint which shows the variation of three parameters: $\mathbf{K}_{\mathrm{R}}, \mathrm{y} \mathbf{B}_{\text {ef }}$ and $\mathrm{a}$ disturbance that can be modeled.

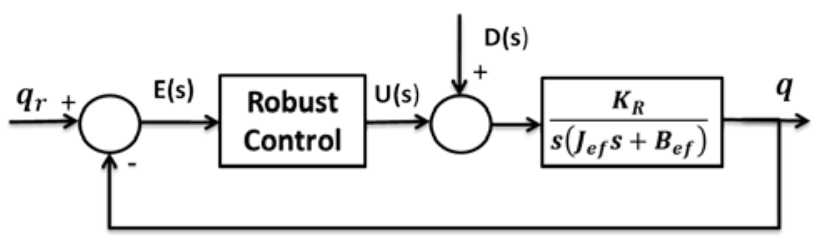

Fig.1. Simplified dynamic model.

\section{B. Parametric Identification}

To determine the uncertainty associated with the dynamic models, a step signal was injected to each joint at different operating points. Therefore the uncertain linear matrix transfer function is a below:

$$
P(s)=\left[\begin{array}{ccc}
P_{11}(s, \infty) & 0 & 0 \\
0 & P_{22}(s, \infty) & 0 \\
0 & 0 & P_{33}(s, \infty)
\end{array}\right]
$$

Where:

$$
\begin{aligned}
& P_{11}=\frac{q_{1}}{u_{1}}=\frac{K_{11}}{s\left(T_{1} s+1\right)} ; \\
& K_{11} \in[0.825-0.975] ; T_{1} \in[0.05-0.4] \\
& P_{2 \mathrm{z}}=\frac{q_{\mathrm{z}}}{u_{\mathrm{z}}}=\frac{K_{2 \mathrm{z}}}{s\left(T_{\mathrm{z}} s+1\right)} \\
& K_{2 \mathrm{z}} \in[0.48-0.75] ; T_{\mathbf{z}} \in[0.05-0.2] \\
& P_{3 a}=\frac{q_{a}}{u_{a}}=\frac{K_{3 a}}{s\left(T_{a} s+1\right)} ; \\
& K_{3 \mathrm{~g}} \in[1.035-1.665] ; T_{\mathrm{g}} \in[0.04-0.2]
\end{aligned}
$$

\section{CONTROLlers Design}

\section{A. $H_{\infty}$ Controllers Design}

The controllers design method $\mathbf{H}_{\infty}$ is related to the minimization of the value peak in the frequency response of some function in closed-loop [10]. F or such purpose the weighting functions $W_{i}$ is introduced in the system to reflect the design goals and also the knowledge that has input and output signals. These signals will be bounded because in the calculation of the robust controller $\mathrm{H}_{\infty}$. the $\|\cdot\|_{\downarrow^{\infty}}$ norm of each signal has its upper limit unit [11].

The inclusion of the weighting functions in a general feedback configuration can be seen in Figure 2. As can be seen the input signals are respectively the signal reference (r), noise (n) and disturbance to the output (d); and the

$$
\left(J_{m}+R^{2} \cdot M(q)\right) * q+\left(B_{m}+R^{2} \cdot V(q, q)\right) * q+R^{2} \cdot F(q)+R^{2} \cdot G(q)+R^{2} \cdot \tau_{p} \quad(6)=K_{m} \cdot R \cdot u
$$


weighted outputs of the system are $\mathbf{z}_{\mathbf{S}}, \mathbf{z}_{\mathbf{U}}$ and $\mathbf{z}_{\mathbf{T}}$.

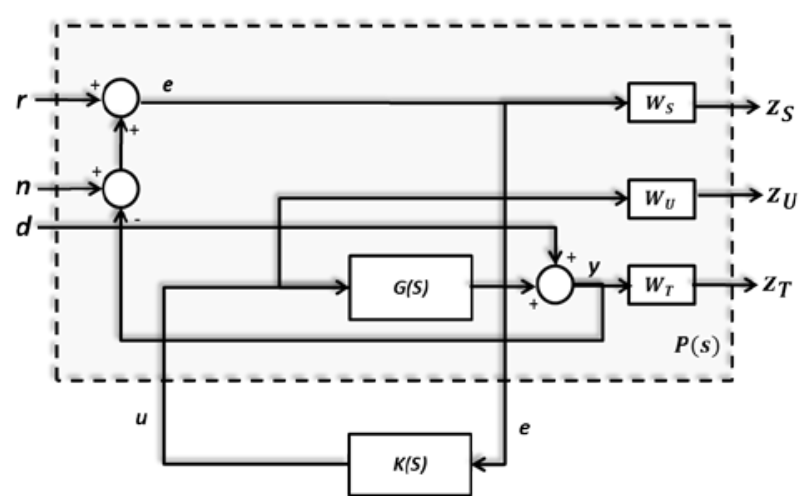

Fig. 2. General configurations for $\mathbb{E}_{\mathrm{m}}$ control problems

The problem of design a $\mathbf{H}_{\infty}$ controller is to find all admissible compensators $\mathbf{K}_{\infty}(\mathbf{s})$ to stabilize internally the system and minimize the norm:

$$
\left\|\mathrm{T}_{\mathrm{zw}}\right\|_{\infty}=\operatorname{Sup}_{\mathrm{w}} \bar{\sigma}\left[\mathrm{T}_{\mathrm{zw}}\right]
$$

The function $W_{\mathrm{T}}(\mathrm{s})$ is used to make robust the system against the uncertainties presented by the inaccuracy of the linearized model and variation of the parameters of the plant. It usually takes low values at low frequencies and high values at high frequency values. To ensure a bandwidth of around $26 \mathrm{rad} / \mathrm{s}$ and provide good robust stability control system, the following weighting function is chosen:

$$
W_{T}(s)=\frac{1.3 *(1+s / 20)(1+s / 50)(1+s / 80)}{(1+s / 120)^{3}}
$$

The function $W_{\mathrm{S}}(\mathrm{s})$ provides an adequate attenuation for perturbations of low frequency and a precise monitoring of the step slogans. Modeling errors and the bandwidth of the actuators generally impose this weighting function to take low values at high frequency.

To ensure a phase margin greater than $41^{\circ}$ and a gain margin above $17.65 \mathrm{~dB}$ the following weighting function is selected:

$$
W_{S}(s)=\frac{\sqrt{0.5} s+15.1}{s+15.1 * 10^{-4}}
$$

The function $W_{\mathrm{U}}(\mathrm{s})$ is intended to reduce the over oscillation of temporal response affecting the speed of the same. Likewise, the inclusion of $W_{W}$ allows avoiding numerical problems in the calculation of the controller. However, $W_{U}=1$ can present excellent results.

\section{B. QFT Controllers Design}

QFT is a control strategy that explicitly proposes the use of feedback to reduce the effects of the uncertainty of the plant and meet the desired performance specifications (Figure 3). This method is quantitative since it a llows designing a controller for the precise amount of uncertainty estimated of the plant, for a given set of perturbations and specifications.

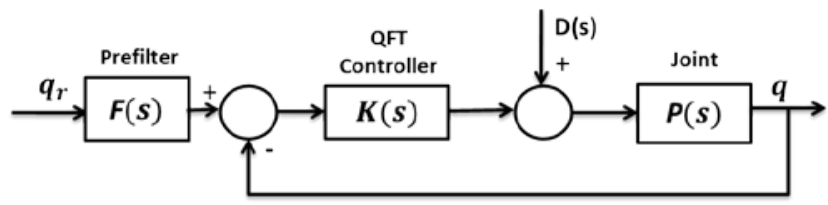

Figure 3. Diagram of QFT Control.

The specifications for the design of controllers for joints were:

Robust Stability:

$\frac{q(s)}{q_{r} F(s)}=\left|\frac{P(s) K(s)}{1+P(s) K(s)}\right| \leq 1.3$

Tracking Performance:

$T_{L}(s) \leq\left|\frac{F(s) P(s) K(s)}{1+P(s) K(s)}\right| \leq T_{U}(s)$

Where

$T_{L}(s)=\frac{200}{(s+4)(s+5)(s+20)^{\prime}} ; \quad T_{U}(s)=\frac{3.2(s+20)}{s^{2}+8 s+64}$

Fig. 4 shows plant uncertainty in Nichols chart for the first link and Figure 5 depicts the robust stability bounds at this frr

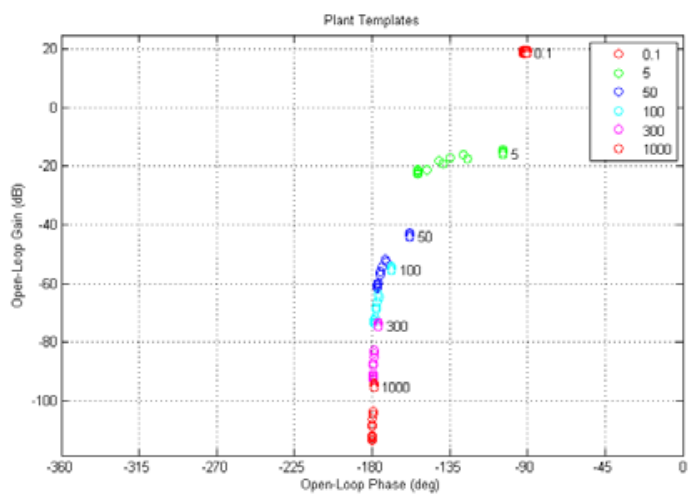

Fig. 4. Uncertainty Templates for Arm 1. 


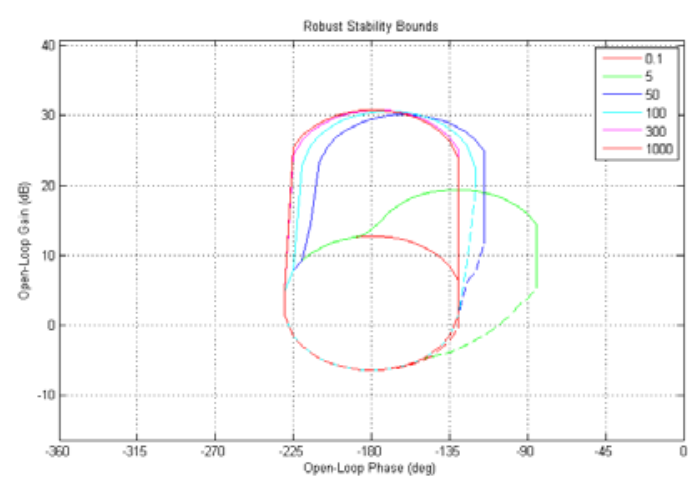

Fig. 5. Robust stability bounds for Arm1.

The overshoot and the setting time specifications $\left(\mathrm{M}_{\mathrm{p}}=5 \%\right.$ and $\mathrm{T}_{\mathrm{s}}=1 \mathrm{~s}$ ) are given in the form of upper and lower bounds in frequency domain, usually based on simple second-order models to represent the status of damped condition (See Fig. 6)

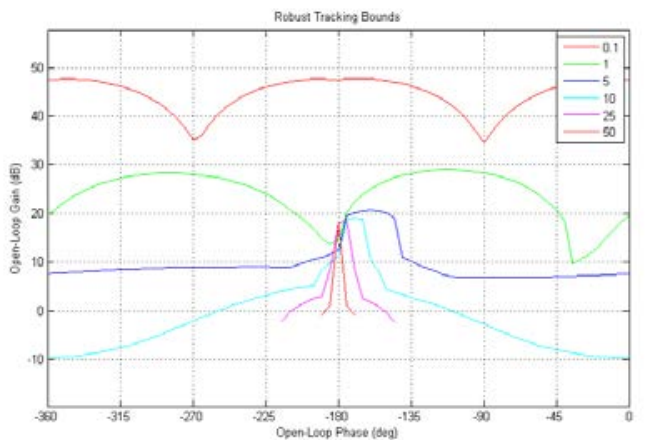

Fig. 6. Robust tracking stability for Link 1

The design of pre-filter guarantees the satisfaction of tracking specification. In Fig. 7 pre-filter shaping of open loop transfer function for the first link is shown.

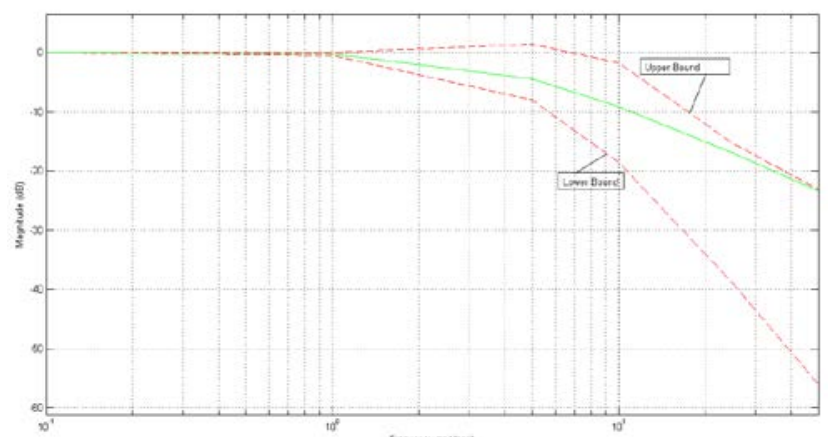

Fig. 7. Pre-Filter Shaping for Link 1

\section{Simulation}

In order to verify the validity of these both kinds of control algorithm, those are put forward by this paper.

\section{A. $H_{\infty}$ Controller}

Based on the weighting functions presented earlier in this paper, $\mathrm{H}_{\infty}$ controllers obtained were as follows:

Link 1:
$K_{1}(s)=\frac{108.9 s^{3}+10^{4} s^{2}+3.22 * 10^{4} s+2.067 * 10^{-6}}{s^{4}+112.9 s^{3}+2284 s^{2}+1.292 * 10^{4} s+19.51}$

Link 2:

$K_{2}(s)=\frac{5.426 s^{3}+688.6 s^{2}+646310^{4} s+5.887 * 10^{-5}}{s^{4}+129.7 s^{2}+1533 s^{2}+3540 s+1.618}$

Link 3:

$$
K_{\mathrm{a}}(s)=\frac{18.19 s^{3}+2201 s^{2}+2.06 * 10^{4} s+2.675 * 10^{-3}}{s^{4}+132.3 s^{2}+2415 s^{2}+1.465 * 10^{4} s+6.701}
$$

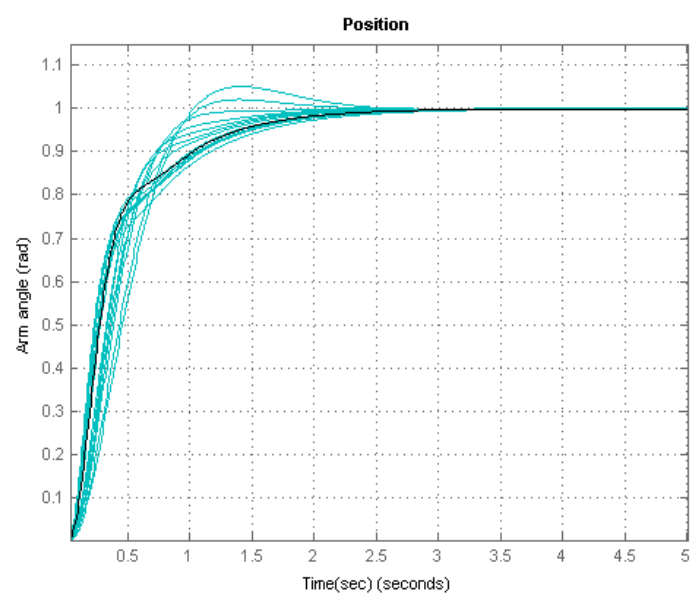

Figure 8 . Tracking stability for Link 1 ( $H_{m}$ Controller)

\section{B. QFT Controllers}

PID controllers were obtained from QFT robust control methodology. The controller transfer function for the articulation QFT $i$ is:

$K_{\tilde{i}}(s)=K_{p}+\frac{K_{I}}{s}+K_{D} s$

And the first order pre-filter associated with this controller is given by:

$$
F_{i}(s)=\frac{a}{s+a}
$$

Table I lists the parameter of PID controllers and the prefilter for each joint, obtained by QFT, and in Fig. 9 the response of the joint 1 is shown.

TABLE I

PARAMETERS OF ADVANCED PID-CONTROLLERS

\begin{tabular}{lcccc}
\hline \hline Link & $K_{P}$ & $K_{I}$ & $K_{D}$ & $a$ \\
\hline Link 1 & 43.5 & 8.5 & 5 & 3.815 \\
Link 2 & 70 & 20 & 5 & 3.0 \\
Link 3 & 31.6874 & 11 & 1.76 & 3.1 \\
\hline \hline
\end{tabular}

The stability margin validation-curve and the tracking performance validation-curve are shown respectively in Fig. 10 and 11. 


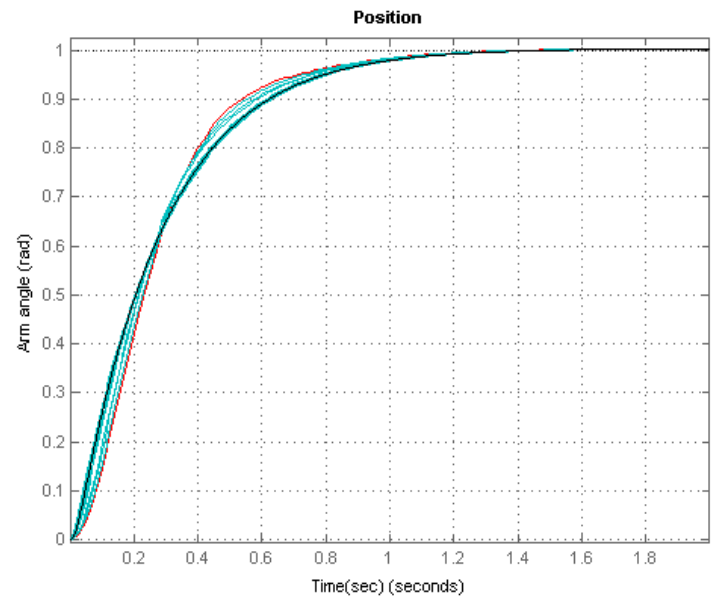

Fig. 9. Tracking stability for Link 1 (QFT Controller)

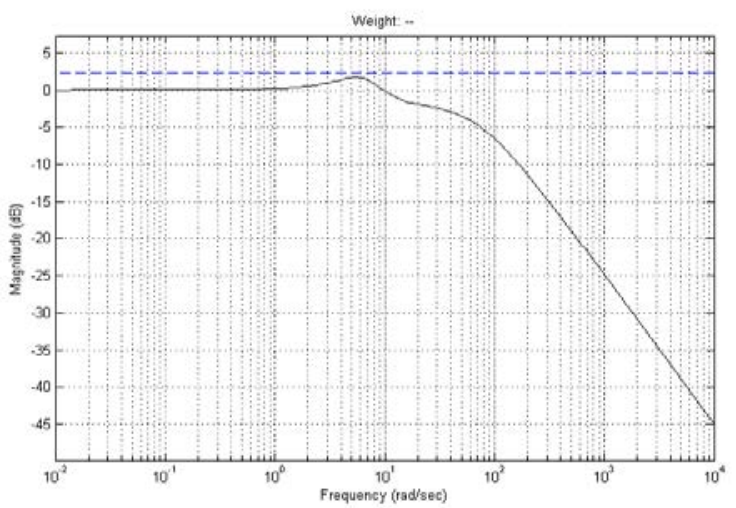

Fig. 10. Stability margin validation-curve for link 1.

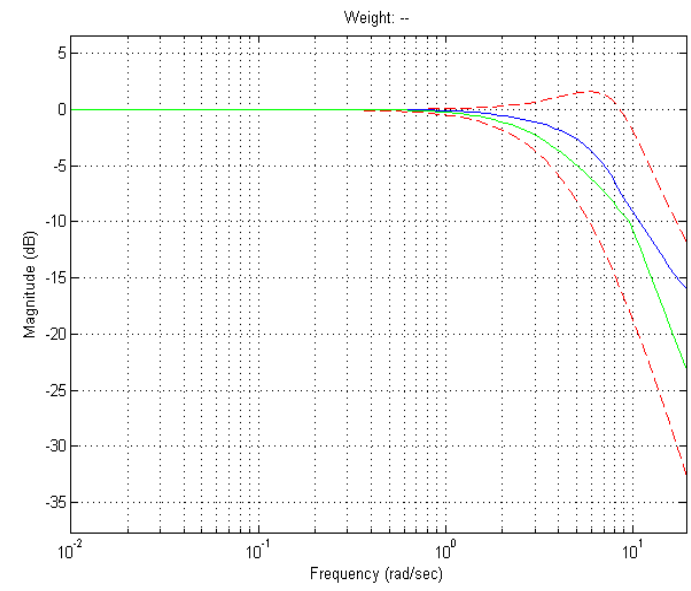

Fig. 11. Tracking performance validation-curve for link 1.

\section{Performance Indices}

In several automatic control applications such as adaptive systems, optimization of parameters and optimal control design, the quantitative measurement of control system performance becomes necessary. The performance indices are quantitative measures of the control system that compares the quality of the controller action and the effort necessary to achieve control.

For our analysis the indices ISE, IAE and ITAE is calculated into four operating points for each joint of the robot in order to compare the performance of $H_{\mathrm{m}}$ and QFT controllers designed. Table II shows the results for $H_{m}$ controllers while the results of QFT controllers are shown in Table III.

TABLE II

PERFORMANCE INDICES FOR $\mathrm{H}_{\infty}$ CONTROLLERS

\begin{tabular}{|c|c|c|c|c|}
\hline Arm & Parameters & ISE & IAE & ITAE \\
\hline \multirow{4}{*}{ Link 1} & $K_{\mathrm{P}}=0.825_{y} T_{f}=0.05$ & 0.2921 & 0.5630 & 0.3660 \\
\hline & $K_{\mathbb{P}}=0.825_{v} T_{F}=0.4$ & 0.4532 & 0.6653 & 0.3591 \\
\hline & $K_{p}=0.975 x T_{p}=0.05$ & 0.2536 & 0.4765 & 0.2697 \\
\hline & $K_{\mathrm{p}}=0.975 \mathrm{x} T_{\mathrm{p}}=0.4$ & 0.4142 & 0.6200 & 0.3261 \\
\hline \multirow{4}{*}{ Link 2} & $K_{\mathrm{D}}=0.48 \mathrm{y} T_{F}=0.05$ & 0.4815 & 0.9665 & 1.0070 \\
\hline & $K_{p}=0.48 ; T_{f}=0.2$ & 0.5527 & 0.9667 & 0.8651 \\
\hline & $K_{\mathrm{p}}=0.75 \mathrm{x} T_{F}=0.05$ & 0.3177 & 0.6192 & 0.4363 \\
\hline & $K_{p}=0.75 x T_{f}=0.2$ & 0.3889 & 0.6194 & 0.3450 \\
\hline \multirow{4}{*}{ Link 3} & $K_{p}=1.035 x T_{f}=0.04$ & 0.2364 & 0.4489 & 0.2465 \\
\hline & $K_{\mathrm{W}}=1.035_{p} T_{F}=0.20$ & 0.3142 & 0.4490 & 0.1758 \\
\hline & $K_{\mathrm{P}}=1.665 T_{\mathrm{F}}=0.04$ & 0.2553 & 0.4182 & 0.1882 \\
\hline & $K_{p}=1.665 T_{f}=0.20$ & 0.1682 & 0.2797 & 0.1075 \\
\hline
\end{tabular}

TABLE III

PERFORMANCE INDICES FOR QFT CONTROLLERS

\begin{tabular}{|c|c|c|c|c|}
\hline Arm & Parameters & ISE & IAE & ITAE \\
\hline \multirow{4}{*}{ Link 1} & $K_{p}=0.825 x T_{p}=0.05$ & 0.0010 & 0.0441 & 0.0942 \\
\hline & $R_{\mathbb{P}}=0.825 x T_{F}=0.4$ & 0.0051 & 0.0665 & 0.0948 \\
\hline & $K_{\mathrm{D}}=0.975 x T_{F}=0.05$ & 0.0007 & 0.0373 & 0.0795 \\
\hline & $R_{\mathrm{p}}=0.975 i T_{p}=0.4$ & 0.0038 & 0.0555 & 0.0795 \\
\hline \multirow{4}{*}{ Link 2} & $R_{\mathrm{D}}=0.48, T_{P}=0.05$ & 0.0028 & 0.0758 & 0.1636 \\
\hline & $K_{\mathrm{p}}=0.48, T_{\mathrm{P}}=0.20$ & 0.0055 & 0.0802 & 0.1579 \\
\hline & $K_{\mathrm{D}}=0.75_{\mathrm{y}} T_{P}=0.05$ & 0.0012 & 0.0485 & 0.1038 \\
\hline & $R_{p}=0.75, I_{p}=0.20$ & 0.0024 & 0.0508 & 0.1000 \\
\hline \multirow{4}{*}{ Link 3} & $K_{\mathrm{D}}=1.035 ; T_{F}=0.04$ & 0.0006 & 0.0351 & 0.0751 \\
\hline & $K_{\mathrm{D}}=1.035 ; T_{F}=0.2$ & 0.0014 & 0.0719 & 0.0367 \\
\hline & $K_{\mathrm{D}}=1.665 \mathrm{x} T_{\mathrm{F}}=0.04$ & 0.0006 & 0.0227 & 0.0444 \\
\hline & $R_{\mathrm{p}}=1.665 \mathrm{~s} T_{p}=0.2$ & 0.0002 & 0.0218 & 0.0465 \\
\hline
\end{tabular}


The simulation results show that the PID controllers designed using QFT approach presented better results than robust $\mathrm{H}_{\infty}$ controllers.

\section{CONCLUSION}

This paper has developed the control system for a manipulator with parametric uncertainty via $\mathrm{H}_{\infty}$ control approach and the design of advanced PID controllers from QFT approach. Although both controllers showed satisfactory results, the simulation results showed better dynamic performance by PID controllers.

\section{ACKNOWLEDGMENT}

The authors would like to acknowledge the financial support provided by the Department of Research and Extension Faculty (DIEF, for its acronym in Spanish), of Universidad Industrial de Santander, Colombia.

\section{REFERENCES}

[1] R. Kelly, V. Santibañez, and A. Loria. Control of Robot Manipulators in Joint Space. Springer, 2005.

[2] Horowitz, P. I. Quantitative feedback theory. Control Theory and Applications, IEE Proceedings D. Vol.129, Page(s): 215 - 226, 1982.

[3] G. Zames, "Feedback and Optimal Sensitivity: Model Reference Transformations, Multiplicative Seminorms, and Approximate Inverses", IEEE Trans. Aut. Contr., Vol. AC-26, pp. 301-319, 1981

[4] M. García-Sanz, C. Houpis, and S. Rasmussen, Quantitative Feedback Theory. Fundamentals and Applications. New York: CRC Press, 2 Edition, 2006, ch. 1-3.

[5] B. Chen, Y. Chang, and T. Lee, "Adaptative Control in Robotic Systems with $\mathrm{H}_{\infty}$ Tracking Performance," Automatica, Vol. 33, No. 2 , pp. 227-234, 1997.

[6] Y. Zuo, and Y. Wan. "Robust $\mathrm{H}_{\infty}$ intelligent tracking control for robot manipulators". IEEE, Proceedings of the 7 th World Congress on Intelligent Control and Automation June 25 - 27, 2008, Chongqing, China.

[7] G. Wil, N. Sepehri y K. Ziaei, "Design of a hydraulic force motion control system using a generalized predictive control algorithm”, In IEE Proc. On Control Theory and Applications vol 5, pages. 428-436. 1998.

[8] B. Yao, F. Bu, J. Reedy y G. Chiu, “Adaptive Robust Motion Control of Single-Rod Hydraulic Actuators: Theory and Experiments" In IEEE/ASME Trans. On Mechatronics, vol 5, pages. 79-91. 2000.

[9] A. Alleyne, R. Liu y H. Wright, "On the limitation of force tracking control for hydraulic active suspensions", In Proc. of the American Control Conf., pages. 43-47. 1998.

[10] R. Rocha, L. S. Martins, "A Multivariable $\mathrm{H}_{\infty}$ Control for Wind Energy Conversion System" IEEE Conference On Control Applications, 2003.

[11] M. Sidi. Design of Robust Control Systems From Classical to Modern Practical Approaches. Krieger Publishing Company, 2001.

\section{Creative Commons Attribution License 4.0} (Attribution 4.0 International, CC BY 4.0)

This article is published under the terms of the Creative Commons Attribution License 4.0 https://creativecommons.org/licenses/by/4.0/deed.en_US 